\title{
Reliable automatic processing of seismic events: solving the Swiss cheese problem
}

\author{
W Törnman Luleå University of Technology, and LKAB, Sweden \\ J Martinsson Luleå University of Technology, and $L K A B$, Sweden
}

\begin{abstract}
BEMIS (Bayesian estimation of mining-induced seismicity) is a fully automatic, near real-time, robust and self-learning seismic processing solution for mining-induced seismic events. A prototype solution is tested in parallel with IMS's routine manual processing in LKAB's underground mines in Malmberget and Kiruna, providing four times more accurate earthquake locations based on 290 known blasts, 40 times faster processing time that scales with computer power, and the ability to detect and locate up to six times more events given the same input data. In addition to a fully automatic system, BEMIS provides a variety of unique functions such as quality control of all results, self-learning adaptation and calibrations, tomography, and prediction models of future seismicity. In this paper, we summarise the results from different investigations throughout time and discuss the unique approach considered to obtain reliable auto-processing in a challenging, unknown and changing environment.
\end{abstract}

Keywords: mining-induced seismicity, automatic processing, statistical seismology, reliable seismic parameters

\section{Introduction}

In seismically active mines, decisions based on seismic data are often essential to continue mining. These decisions often span from short-term evacuation of areas subjected to increased activity or large seismic events, all the way to long-term production and mine design planning with the aim to limit future induced seismicity. The seismic data is also used to understand the effects of mining and stress redistribution on the surrounding rock mass. This can be achieved either by investigating individual, often large events and their seismic parameters and stresses (Sjöberg et al. 2011) or by considering many events in combination with different mining data as covariates in statistical models to infer effects or predict future activity (Martinsson \& Törnman 2019) as well as future environmental impact on residents nearby (Wettainen \& Martinsson 2014). Additionally, many events and their source-sensor rays can be used along with tomographic techniques to visualise the rock's acoustic properties and changes over time.

Mining environments are often very heterogeneous (Martinsson 2012; Martinsson \& Törnman 2019), containing complex geology and excavated volumes that are continuously changing due to further excavation (Nordqvist \& Wimmer 2016; Troll et al. 2019; Vatcher et al. 2015, 2018). A changing environment leads to frequency-dependent and spatiotemporal velocities, attenuations and direction of arrivals, affecting the measured seismic waveforms and their arrivals. In comparison to the study of tectonic movements over larger distances, the measurement system in a mine can be thought of as sensors located in the middle of a Swiss cheese, where the ray paths, velocities, attenuations and direction of first arrivals between the event location and corresponding sensors differs significantly compared to a homogeneous medium, as illustrated in Figure 1. 


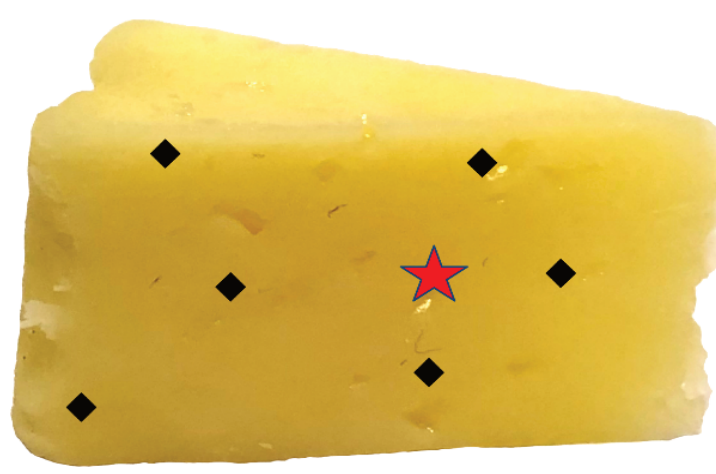

(a)

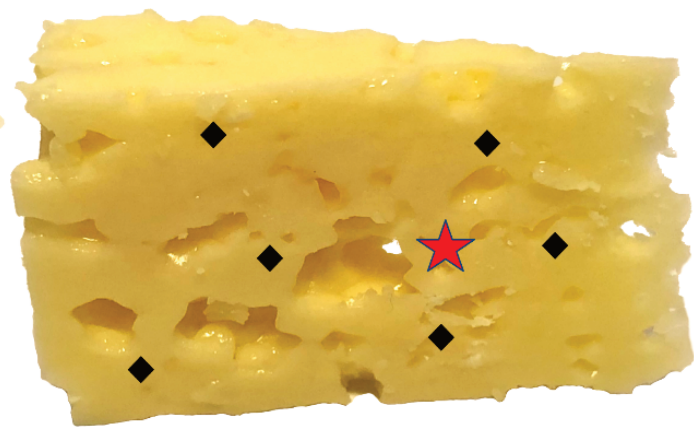

(b)

Figure 1 Illustration of the difficulties of studying mining-induced earthquakes with two different types of propagation medium in relation to the length of the ray paths. (a) We illustrate a measurement system at large distances where the rock mass can be modelled as homogeneous; (b) We have a measurement system in a mine where the rays and waveforms are significantly affected by heterogeneities. This simplification illustrates the effects of cavities but in reality, the acoustic properties in the entire rock mass differs with geology, fractures and stresses

These challenges, denoted here as the 'Swiss cheese problem', are often the main obstacles for obtaining reliable auto-processing of seismic events and today, mines usually resort to manual processing to obtain reasonable hypocentre location and additional seismic parameters. However, addressing these challenges are also difficult for a human processor, needing to adjust for these unknowns with the aim to obtain realistic results. In difficult processing situations this will lead to significant discrepancies of the end result when comparing the processing of different human processors of the same data. We will touch more on this in Section 2.

To obtain reliable results prior to any processing is conducted, we must instead calibrate each sensor to its surrounding rock mass (i.e. compensate for the Swiss cheese). This can be done either by using known events (e.g. blasts) from different locations or by using the seismic data itself and machine learning techniques (e.g. see Martinsson 2012 for a more detailed description of these approaches). For auto-processing in particular, it is also essential to include robust statistics and prior information to handle surrounding noise, outliers, and inconsistent data (Martinsson 2012).

BEMIS (Bayesian estimation of mining-induced seismicity) is a module-based, auto-processing platform for seismic data that addresses the Swiss cheese problem. The modules in the platform are results obtained over a number of years from LKAB's internal research and development, and Luleå University of Technology. The long-term goal with BEMIS is to replace today's manual processing with a fully automatic processing routine that delivers fast, accurate and reliable seismic results as the basis for short- and long-term decisions.

In this paper, we will start by introducing the challenges with manual processing as this is one of the driving forces behind the research and the development of BEMIS. We will then summarise a few interesting results from various investigations throughout time and motivate the approach taken in BEMIS to solve the Swiss cheese problem.

\section{Human limitations}

As with all manual interventions, there are discrepancies between individuals. To process seismic data manually, each processor makes a number of decisions. For example, how to classify the event (e.g. labelling it as a seismic event, a blast, or mining noise), what sensors to include in the processing, how to pick the arrival times, is the end processing result realistic and satisfying or do we need to make adjustments, etc.

In this section, we will highlight a few challenges we encounter and provide some comparisons to the automatic processing solutions in BEMIS. 


\subsection{Data decimation}

Data is often discarded in a number of steps to reduce noise and to make it manually manageable. The first step is in associating triggered waveforms to single events, which is done automatically by the seismic measurement systems in LKAB (Kiruna and Malmberget) and in Boliden (Garpenberg). The sensitivity of the association can often be adjusted by different settings (e.g. minimum number of triggers) and also the triggering levels can be set to limit the number of triggered waveforms. Reducing the sensitivity by these settings means that only significant events are detected and processed, limiting the number of waveforms to be manually processed and classified.

In Malmberget and Kiruna, the settings in the association step discards approximately $85 \%$ of the triggered waveforms, leaving $15 \%$ to be associated with events. Out of the remaining data, approximately $70-85 \%$ of the associated events are then rejected as noise by the routine manual processing applied in Malmberget and Kiruna mines.

After the association step, a manual processor will further decimate the data in the sequential analysis. For example, in the localisation step, a manual processor usually picks and includes a maximum of 18 sensors regardless of how many sensors have been associated to the event, as shown in Figure 2 (both LKAB mines contain more than 200 installed sensors). There are mainly two reasons for this reduction of data: 1) To make it manually manageable; and 2) The first sensors are considered more important in the analysis.

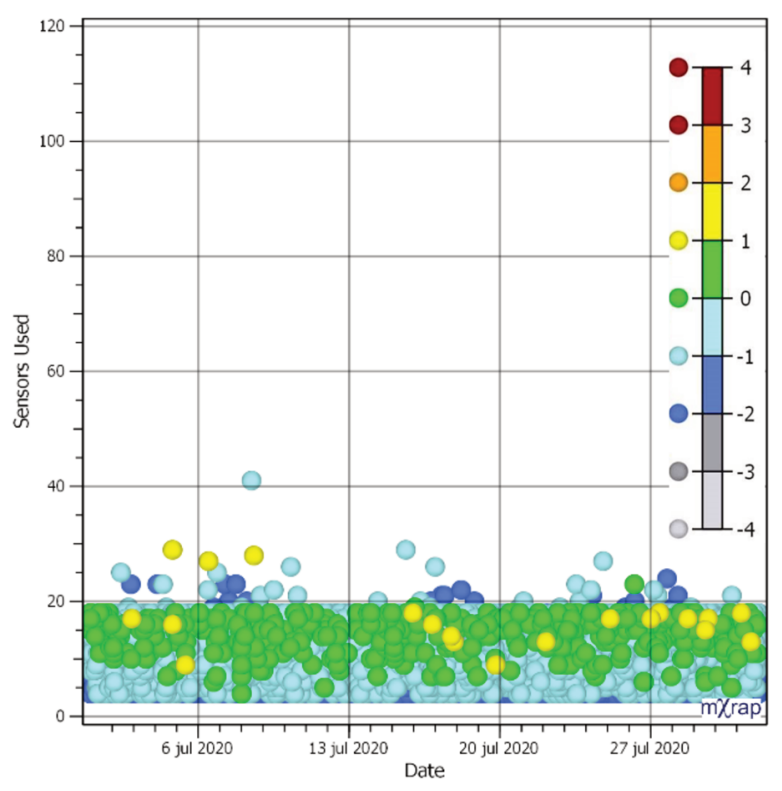

(a)

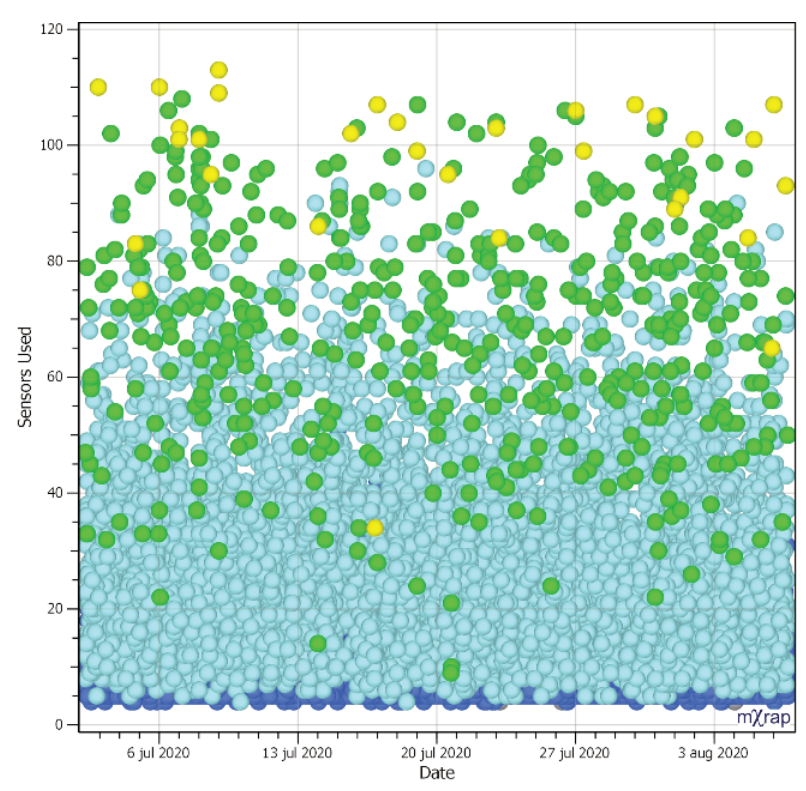

(b)

Figure 2 The dots represent the number of sensors used when processing the event (on the vertical axis) versus the event-times on the horizontal axis. The dots are coloured by event magnitude (according to the colour scale) and both figures are for the same time period in the Malmberget mine: (a) Sensors used in routine manual processing; (b) Sensors used for auto-processing in BEMIS

With limited human resources, these two reasons make sense. However, adopting this criterion may give undesired artefacts such as ambiguity in locating events and biased source parameters. From a hypocentre estimation perspective, it is essential that we solve the Swiss cheese problem as the closest sensors are more affected by the heterogeneities relative to the propagation path, compared to more distant sensors (Martinsson 2012). A good hypocentre estimate depends on good angular sensor coverage, and neglecting distant sensors may cause instabilities like mirroring events commonly observed in Kiruna and described more in Section 2.2. Also, it is important to include a valid uncertainty description in the statistical model to obtain proper weighting with respect to distance (Martinsson 2012). Here, the validity of the uncertainty 
description (or weighting) must be tested using residual analysis (Martinsson 2012) or it may corrupt the sequential analysis. An invalid description often favours a truncation of distant sensors as they may harm the localisation step. A valid description on the other hand weights the information with respect to its uncertainty and can be shown to be optimal (Kay 1993; Pintelon \& Schoukens 2001). Additionally, the closest sensors may violate the far-field assumption and relying only on these for source parameter estimation may be questionable.

Increasing the sensitivity of the measurement system will increase the number of locatable events using the same measurement system. Increased sensitivity will also increase the quality of existing events by incorporating more data, under the condition that we apply robust statistics to handle additional outliers and noise. This is particularly valuable in studying precursors (Nordström et al. 2020), creating prediction models (Martinsson \& Törnman 2019; Wettainen \& Martinsson 2014), or generating tomographic images of the rock mass, all directly depending on the amount and quality of detectable events.

From an auto-processing perspective, computer resources are the limitation. There is generally no need to limit the amount of triggered data or associated events. The association can additionally be done using more advanced arrival time estimation algorithms combined with heterogeneous model assumptions that is calibrated for the Swiss cheese (Martinsson 2012). Today, the association relies on trigger times between sensor pairs along with homogeneous assumptions, where the trigger times are estimated using simple short-time long-time average techniques.

Noise rejection also improves with more data, described more in Section 3.1 and Appendix A, providing more evidence to the classification algorithms. It is beneficial to run all associated events through the entire processing chain, prior to the classification step, as opposed to rejecting the event based only on waveform analysis. The advantage is that the classification algorithms can work with significantly more covariates than just waveform characteristics (e.g. hypocentre estimation quality and spectral parameters describing the source). This approach may also provide an opportunity to classify events in more classes (e.g. orepass noise, different types of blasts, hydraulic hammers, etc.). When conducting manual processing, these events are often left unprocessed due to limited resources. However, in automatic processing all associated events are processed, providing not only more data for improved classification but also additional events that can be used for other applications (e.g. orepass level and volume estimation, blast analysis, tomographic images and general system calibrations).

\subsection{Human dependent results}

Classification of events is a challenging task and is both mine- and site-dependent. Among individuals within a group of processors, there are naturally discrepancies in how events are classified when the processors are subjected to the same dataset. The difference in similarity between individuals in determining if an event is accepted or rejected can be as low as $50 \%$ but is generally around $80 \%$ (see Appendix A and Figure A1 for more details).

The hypocentre location also differs between individuals. In LKAB, the largest events $(m \geq 1)$ are routinely sent for manual reprocessing by a more experienced processor as a precaution. Documenting the relocation after reprocessing we can obtain statistics of the effects of manual reprocessing on the largest events. Here we used data between 26 April 2016 and 13 July 2017 in Kiruna, resulting in 1,209 events with $m \geq 1$ and out of which 506 events had documented coordinates after reprocessing. A histogram of the relocation distance of the 506 events due to reprocessing are shown in Figure 3a, and the movement of each event is shown in Figure $3 \mathrm{~b}$ represented as a line between the dots. 


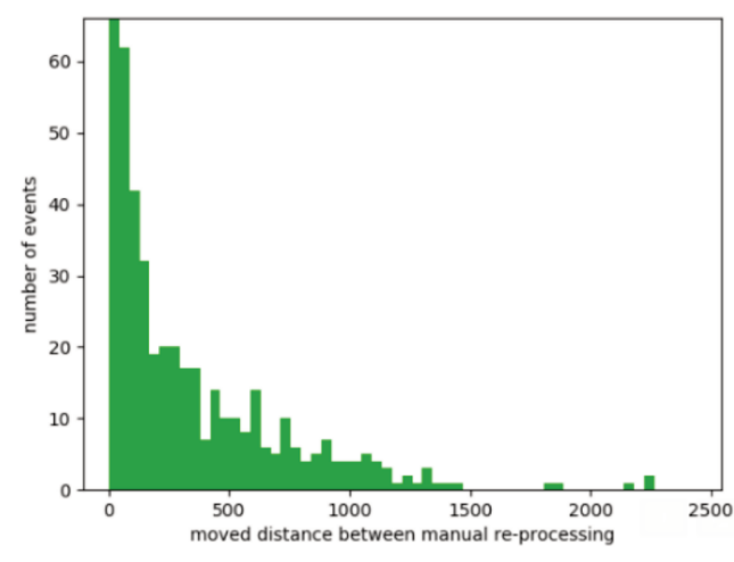

(a)

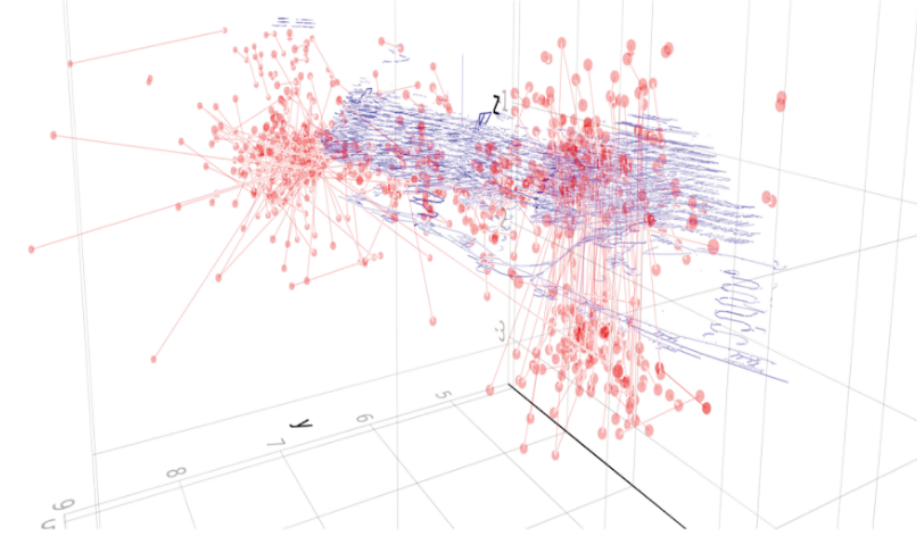

(b)

Figure 3 Shows the relocation of 506 large events $(m \geq 1)$ due to manual reprocessing in Kiruna mine: (a) Histogram of the moved distance due to manual reprocessing; (b) Event locations represented as red dots where the lines between the dots mark the moved distance of an event's coordinates due to reprocessing

We can summarise the result and conclude that the coordinates of a large event in Kiruna moves on average a distance of $322 \mathrm{~m}$ due to reprocessing (with a 95\% confidence interval of 283 to $360 \mathrm{~m}$ ). In Figure $3 \mathrm{~b}$, we can see the difficulty in manually locating large events with only a few sensors as we mentioned in the introduction and showed in Section 2.1. Changing a few arrivals or replacing a few sensors that are used in the hypocentre location procedure may result in a large movement of the event's coordinates. We also see movements of coordinates between different clusters. Many of the larger events in Kiruna are located in the hanging wall where the sensor coverage and configuration are far from optimal. The poor coverage in this area, combined with neglecting sensors further away, contributes to false hypocentres and mirroring effects where two clusters are almost equally likely. Here, a solution to the Swiss cheese problem is especially important as sensors on the footwall will otherwise not compensate for the large excavated regions between the sensors and the event (e.g. visualised in Figure A2 and illustrated in Figure B2). This will result in inconsistent arrivals, extreme values or outliers in the localisation step resulting in very different coordinates for an event depending on which sensors are included in the analysis, as shown in Martinsson (2012).

\subsection{Processing time}

Manual processing time may affect short-term decisions and algorithms acting on precursors to detect anomalies or increased seismic risk. In cases of a sudden increase in activity in the mine, the processing time increases and events are queued due to finite resources. Here, automatic processing can easily scale with computer resources and, if necessary, the computations can use dynamic resource allocation to adjust for the temporary increased load while keeping computational cost low during normal activity. The automatic processing of events in Kiruna and Malmberget takes on average a minute per event and cpu core (e.g. in Malmberget the average compute capacity is 64 events per minute) and the time will decrease further with code optimisations and extended parallelisation in the near future.

\section{$2.4 \quad$ Number of sensors}

Today, LKAB has more than 200 sensors in each underground mine, ranking the systems to be among the largest in the world for detecting mining-induced events. These two networks detect between 10,000 up to 20,000 events per day, and for which only $15-30 \%$ of these are provided with accepted coordinates after manual processing (e.g. 31\% in Kiruna and 13\% in Malmberget between 25 July 2020 and 4 August 2020. Increasing the sensitivity of the measurement system by introducing more sensors will quickly approach the limit of what manual processing is capable of. This is also a consequence of the Gutenberg-Richter law, where there are exponentially more smaller events detectable with a denser network (e.g. Martinsson \& 
Jonsson 2018). Additionally, as the mine goes deeper, the stresses increase and there are observations indicating that the number of events doubles per $100 \mathrm{~m}$ increase in depth. Vallejos \& Mckinnon (2011) reported a factor 1.90 more events for Creighton Deep (or more precisely $0.0001 \exp (0.0064 z)$ where $z$ is the depth in metres) and Martinsson \& Törnman (2019) reported a factor 1.94 for the Malmberget mine (although rather uncertain due to limited data). The conditions mentioned above emphasise the need for fast and accurate automatic processing without human limitations.

\section{Bayesian estimation of mining-induced seismicity}

BEMIS is a module-based processing platform for seismic data. The guidelines used in the development of each module in BEMIS are to apply: 1) robust Bayesian statistics; 2) capture and include the underlying uncertainties; and 3) automatically compensate for systematic errors that deviate from the assumed model (i.e. solve the Swiss cheese problem). Following these three guidelines, the objective is to obtain robust, reliable and precise estimates, respectively, in each module.

To keep realistic descriptions of the uncertainties throughout the entire processing chain, the input to and output from each module in BEMIS are described by probability distributions, rather than values. We also use customised Markov-Chain Monte-Carlo (MCMC) methods for inference (Martinsson 2012), enabling us to work with realistic distributions and non-linear relationships of model parameters at the cost of increased computational complexity and convergence analysis (Kruschke 2014).

In sequential analysis based on BEMIS results, the information delivered can easily be filtered or weighted based on the associated uncertainties with all values. The uncertainties give a measure of estimation quality and we will show a few examples of this below. Without confidence in a value, that value is meaningless and may impair subsequent analysis and conclusions.

\subsection{Classification}

The classification in the current BEMIS platform is defined by several measures (e.g. waveform time-series analysis, volume of the hypocentre distribution, uncertainty in magnitude, etc.). For further analysis, e.g. in mXrap (Harris \& Wesseloo 2015), the more interested users are allowed to adjust the classification criterion from the default settings. For example, to detect seismic structures, it is favourable to only include well-located events (i.e. where the uncertainty volume is small), while studying the activity in an area with poor sensor coverage (see Section 3.2 and Figure 5), it may be interesting to include events where the location is more uncertain.

In Figure 4, we compare the classification of events between BEMIS and routine manual processing (RMP) using a frequency-magnitude chart. The black curve represents the events that BEMIS rejects but RMP accepts. These events have significantly different statistics ( $b$-values) compared to, for example, the events that both BEMIS and RMP accepts in pink. The majority of the events in black are orepass noises based on waveform analysis, size (Figure 4) and spatial (Figure A2) distribution of the events. In sequential analysis, like estimating the seismic hazard within the area, these events should be discarded.

The green curve represents the events that BEMIS accepts but RMP rejects and it follows the same characteristics as the pink curve. This partly explains the difference seen between BEMIS and RMP in Figure 5 where genuine events are lost. 


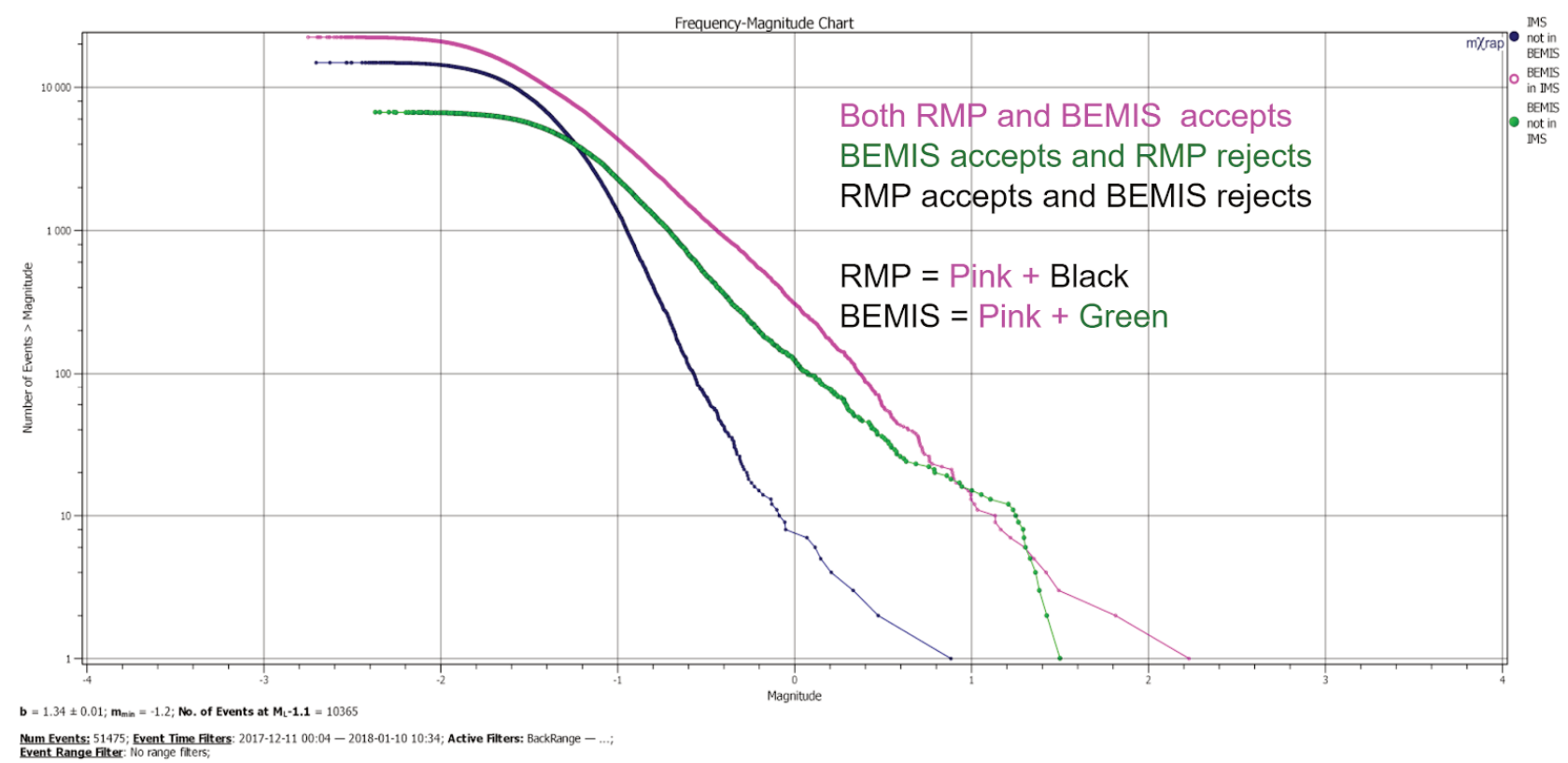

Figure 4 Here we see how Bayesian estimation of mining-induced seismicity (BEMIS) automatically classifies events compared to routine manual processing (RMP) seen from a Gutenberg-Richter perspective. The data shown is for $\mathbf{2 0}$ days in Malmberget using default classification settings in BEMIS. Black curve represents the events that BEMIS rejects but RMP accepts. The green curve represents the events that BEMIS accepts but RMP rejects. The events in the black curve are mainly orepass noise (e.g. Figure A2) and should be discarded to not degrade the quality of seismological analysis of the catalogue.

\subsection{Location and source parameters}

The hypocentre estimation algorithm described in Martinsson (2012) consists of both soft and hard restrictions where the soft restrictions regularise the solution towards realistic and more likely regions when the evidence (i.e. likelihood) is weak. The hard restrictions consist of the extracted rock mass and surface restrictions, forcing the estimates to occur in the rock mass. In both LKAB mines, these restriction models (i.e. prior distributions) are estimated automatically. We use previous seismicity for the soft restrictions to model the seismically active regions, and production blasts and surface topology map for the hard restrictions.

Using a prior distribution in BEMIS is optional but highly recommended. The prior distribution captures our prior beliefs and is particularly useful when we have inconsistent or too few arrivals to form a single conclusion (i.e. a unique hypocentre), as shown in Martinsson (2012). It can be shown that if the prior information is correct, the Bayesian solution is optimal on average (Kay 1993). Here we must emphasise that our prior distribution is a model based on recent observations and is never perfect, however, a model that captures the main features of the seismically active regions is considerably better (from a mean square error perspective) than neglecting prior information and resorting to flat prior distributions where events equally likely can occur anywhere.

An advantage of applying robust Bayesian statistics is that we increase the sensitivity of the measurement system without adding more sensors, simply by providing algorithms that can locate events with far less arrivals or events containing outliers. In Figure 5, we see a comparison in Malmberget, during the same time period, between the RMP and automatic processing using robust Bayesian statistics in BEMIS. In areas with low sensor coverage, BEMIS can locate up to six times more events using the same data. We also see the effects of neglecting prior information in Figure 5a, e.g. where events are located above the surface and within extracted rock mass. 


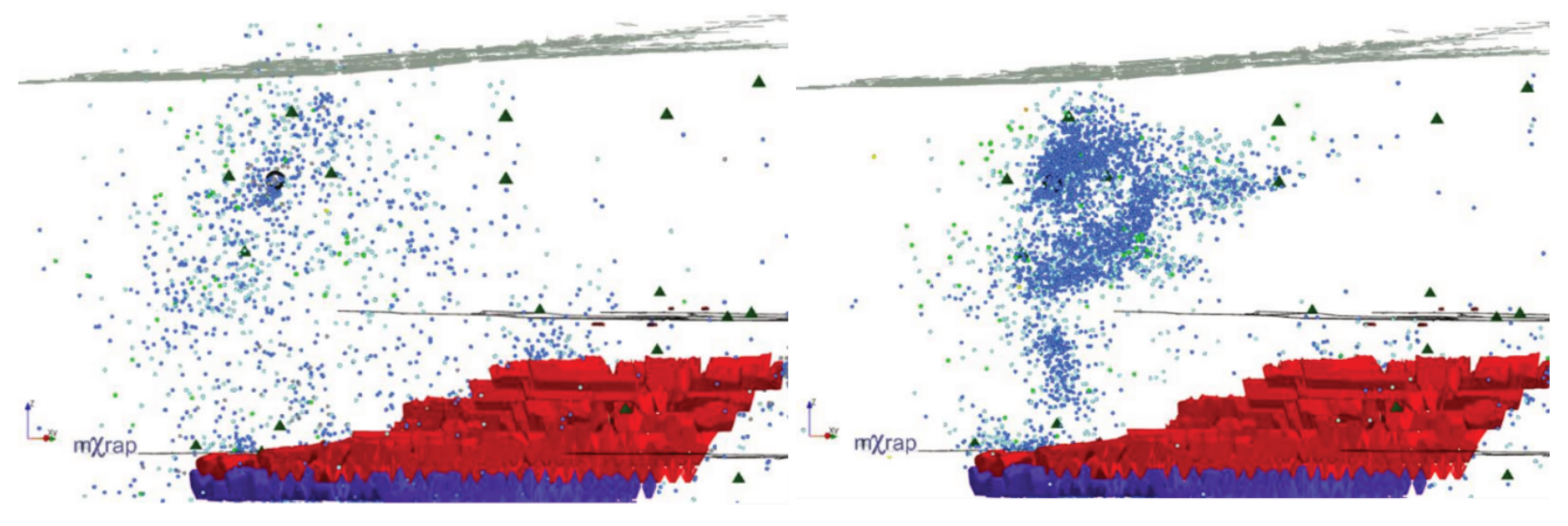

(a)

(b)

Figure 5 A comparison in Malmberget of located events between: (a) Routine manual processing; (b) BEMIS auto-processing. The grey surveys are the surface map and blue and red surveys correspond to the orebody and extracted rock mass, respectively. The dots correspond to the event locations and are coloured by magnitude. Black triangles are the sensors

The algorithm has been evaluated using blasts with known coordinates (Martinsson 2012) and is currently running in parallel with RMP since 2015 in Malmberget and since 2017 in Kiruna. Additionally, off-line batch processing has been conducted of the seismic data from Boliden's mine in Garpenberg (Sweden). Here we will present some of the findings explaining the strengths of using BEMIS and comparisons to RMP.

In Table 1, a comparison of the location accuracy and precision between RMP and BEMIS automatic processing is given, and the results are based on 290 blasts with known coordinates. Using BEMIS reduces the location errors by a factor 3.7 in Kiruna (based on 229 blasts) and by a factor 4.6 in Malmberget (based on 61 blasts). Additionally, the standard deviations of the location errors are reduced by a factor 7.0 in Kiruna and a factor 4.8 in Malmberget indicating a significant improvement in hypocentre precision (in a least-squares sense).

Table 1 Comparison of the location accuracy and precision using routine manual processing (RMP) and BEMIS automatic processing based on 290 blasts (between year 2008 to 2017) with known coordinates out of which 229 are in the Kiruna mine and 61 are in the Malmberget mine

\begin{tabular}{lll}
\hline & $\begin{array}{l}\text { Kiruna 229 blasts } \\
\text { RMP/BEMIS }\end{array}$ & $\begin{array}{l}\text { Malmberget } 61 \text { blasts } \\
\text { RMP/BEMIS }\end{array}$ \\
\hline Mean location error $(\mathrm{m}):$ & $51.8 / 13.9$ & $48.3 / 10.6$ \\
Standard deviation of location error $(\mathrm{m}):$ & $140.2 / 19.9$ & $54.2 / 11.3$ \\
\hline
\end{tabular}

The inclusion of uncertainties in arrival times, velocities, and ray paths, is naturally propagated to the hypocentre estimates using MCMC sampling methods (Martinsson 2012). This approach in combination with sampling algorithms provides realistic uncertainty estimates that depend on all the involved uncertainties as well as the configuration of the sensors (Martinsson 2012). Here, $95 \%$ of the known blasts are within the $95 \%$ uncertainty region (Martinsson 2012). In Figure 6, we see an example of the uncertainty region visualised in mXrap. 


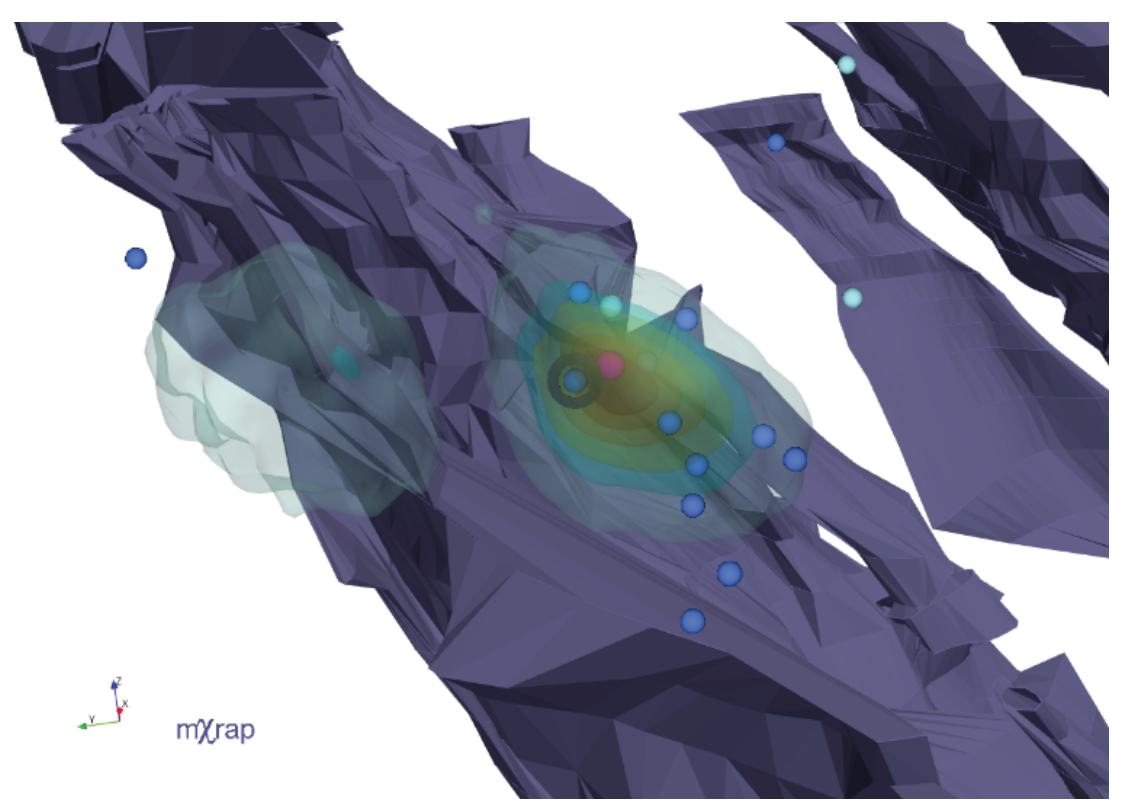

Figure 6 Example of a hypocentre distribution for an event in Malmberget and shown here in $m X$ rap. The hypocentre distribution is represented by the most probable coordinate (the pink sphere) and different degrees of location uncertainty as volume contours in different colours. The uncertainty volume appears automatically in $\mathrm{mXrap}$ by clicking on the event marker

A solution to the Swiss cheese problem is essential in locating events, whether processing is done manually or automatically. In the location algorithm, a solution can be obtained by calibration events with known locations (such as blasts or mechanically induced events), or the surrounding seismicity itself, or both (see Martinsson 2012 for more details). The benefits of using the surrounding seismicity is that calibration can be conducted automatically without any additional resources and it will also give us significantly more calibration points. Additionally, calibrations based on the surrounding seismicity is not restricted to the mine's infrastructure where specific calibration events can be conducted, and consequently we obtain calibrations from the most important regions where we have induced seismicity.

In BEMIS, we continuously calibrate each sensor for the Swiss cheese and corresponding changes, and new sensors that are installed follow the same approach. These calibrations are all spatiotemporal and include, for example, velocities, angle of first arrivals, and size and frequency-dependent attenuations. These are all needed to calibrate for the heterogeneities (i.e. the Swiss cheese) when estimating parameters such as hypocentre, moment tensors, energies and dynamic source parameters. We visualise some interesting calibration data for a few sensors in Appendix B.

Source parameters like seismic moment, source radius, and apparent stress are of importance for the mine and necessary in determining the physics of the event. These parameters are usually derived from the spectral parameters $\left(\Omega_{0}, f_{c}\right)$ in Brune's model (Brune 1970). However, the estimated parameters are commonly associated with large uncertainties and this is especially true in mines where the data are subjected to near-field effects, ambient noises, as well as large path and size dependent variations in the rock mass. These effects are difficult to physically model but they can be compensated for in BEMIS by solving the Swiss cheese problem. In Figure 7, we see an example of this in BEMIS when estimating the spectral parameters $\left(\Omega_{0}, f_{c}\right)$ for an arbitrary event in Kiruna. The plots in the rightmost column are the observed spectra in cyan, blue and magenta for closest sensor, middle sensor and furthest sensor from source, respectively. The observed spectra are shown together with the modelled spectra with and without a solution to the Swiss cheese problem in black and red, respectively.

We clearly see the effect of solving the Swiss cheese problem as the model in black captures both heterogeneities and site effects. The reduced modelling errors leads to improved precision compared to the homogeneous model shown in red. 


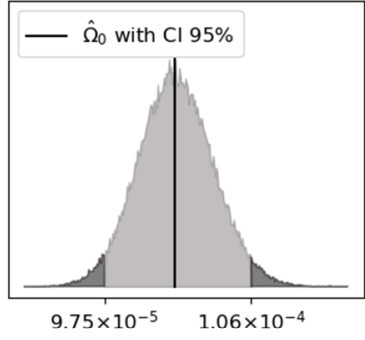

$\hat{\Omega}_{0}$
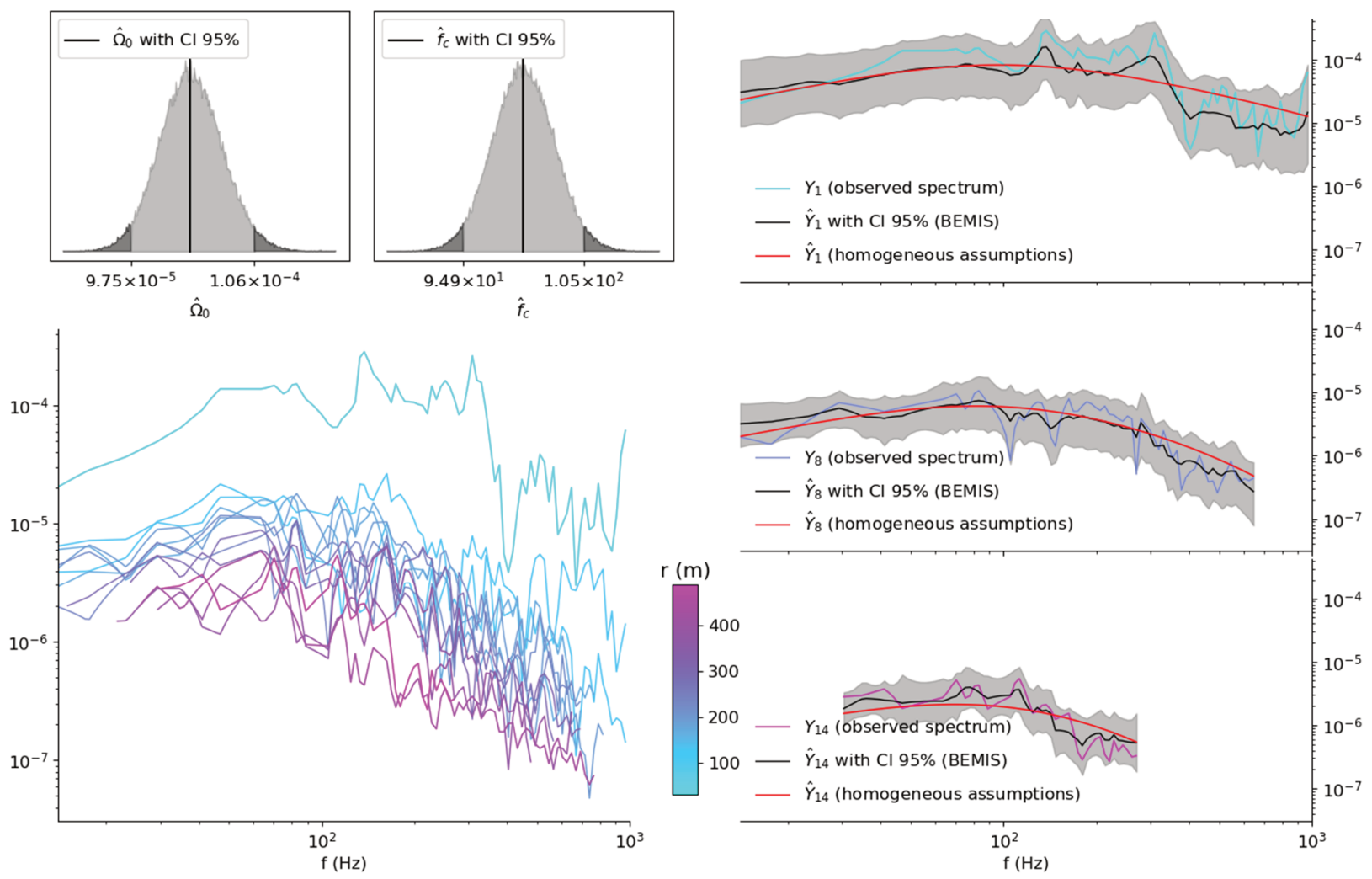

Figure 7 Bayesian inference of the underlying parameters $\left(\Omega_{0}, f_{c}\right)$ in Brunes's model (Brune 1970) to determine the source parameters (e.g. seismic moment, seismic energy, and apparent stress) for one arbitrary event. The lower left corner depicts the observed site spectra coloured by ray-length. The plots in the rightmost column are the observed spectra in cyan, blue and magenta for closest sensor, middle sensor and furthest sensor from source, respectively, and are shown together with the modelled spectra in red using homogeneous assumption and in black (with corresponding $95 \%$ uncertainty) using BEMIS. The solution to the Swiss cheese problem is clearly visible as frequency-dependent resonances in black resemble the observed spectra and depart significantly from the homogeneous assumptions provided in red

\subsection{Prediction models}

The increased number of locatable events (Figure 5) in combination with quality measures on all parameters (Figures 6 and 7) given by BEMIS auto-processing, provides a unique opportunity for robust prediction of future seismicity (Martinsson \& Törnman 2019). In Figure 8, an example of weekly predictions of the activity is presented for three orebodies in Malmberget, capturing the observed activity. We also see exponential decays of the activity when mining stops and accumulation when mining continues. These decay times can be expressed as seismic half-lives (Martinsson \& Törnman 2019) and is defined here as the amount of time required for the activity to fall (or rise) half ways to its steady-state value, if we would decrease (or increase) the production by a certain amount at present time. Interestingly, the half-lives for the seven orebodies in the study ranges from two weeks up to 10 weeks (Martinsson \& Törnman 2019). 


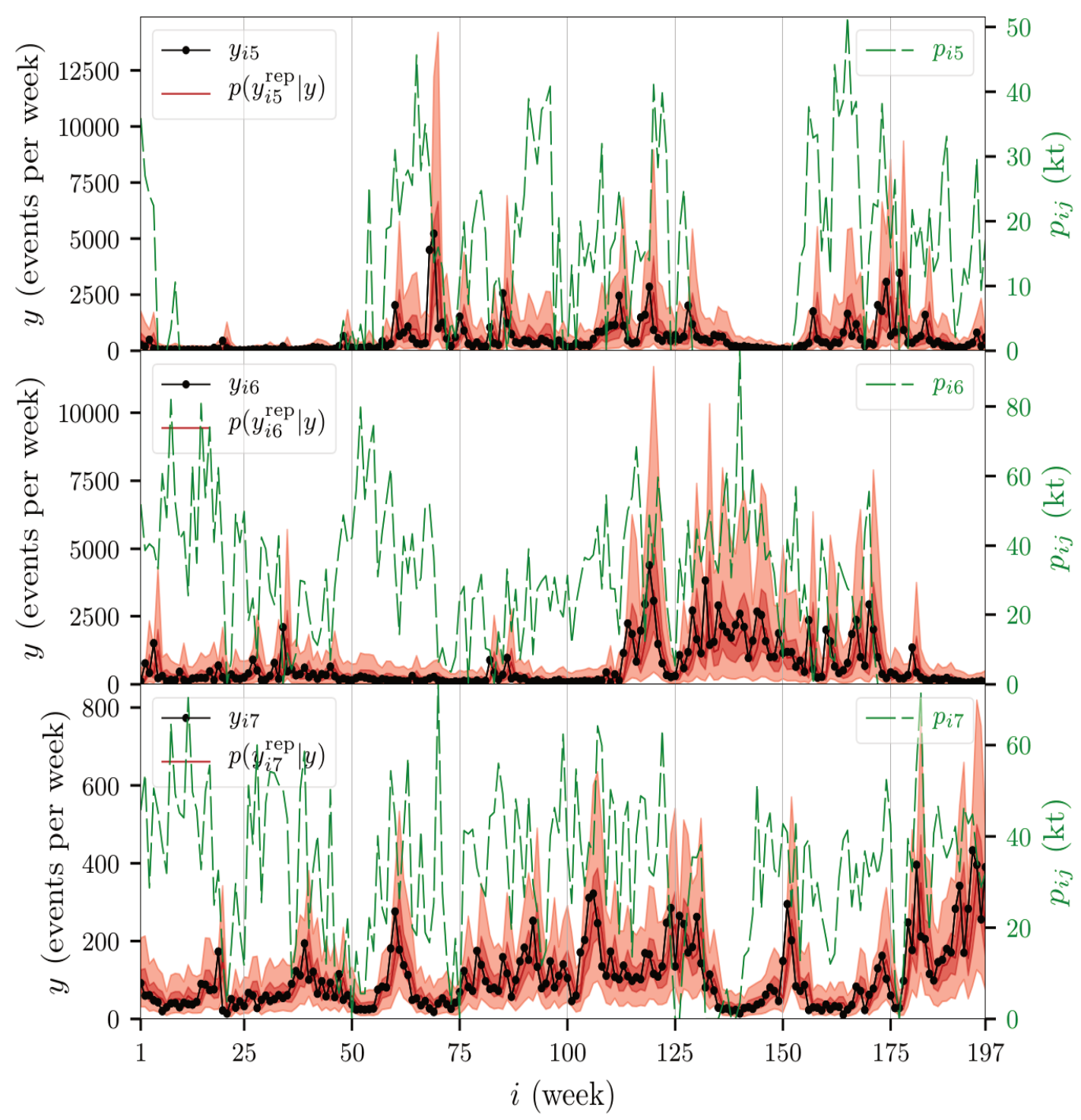

Figure 8 Weekly predictions of the induced activity in the three out of seven orebodies (from Martinsson \& Törnman 2019). The predictions are compared against the actual observations shown as black dots. The green dashed curve represents the planned weekly accumulative rock mass retrieved by the planned production blasts in the orebody, and is used as a predictor in the model. The red curve shows the median prediction and the light red and dark red regions are the $50 \%$ and $95 \%$ prediction intervals, respectively

The activity is one important piece of information in customising production plans and gives an indication of the seismic response to production. However, it does not tell us about the size of the events. In Figure 9, we generalised the Gutenberg-Richter relationship to incorporate the measurement system and spatiotemporal variations of both the system's sensitivity as well as the seismic activity (Martinsson \& Jonsson 2018). This is important as spatiotemporal variations are common due to the excavation being the driving force combined with local changes and expansions of the measurement system. 


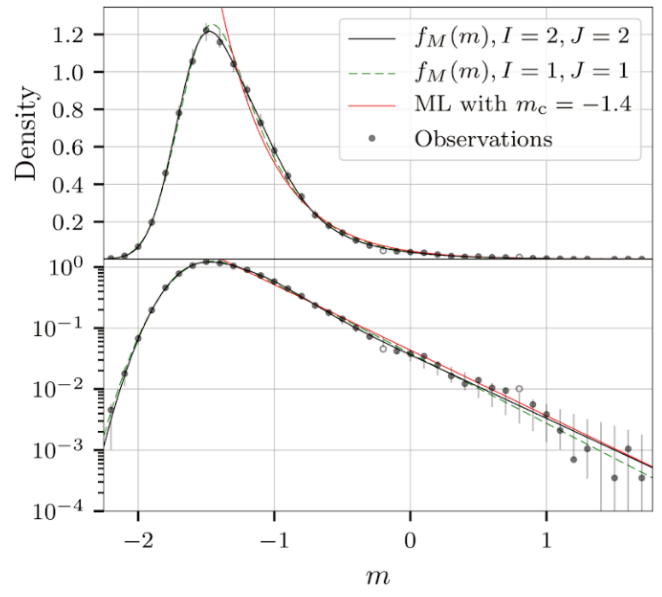

(a)

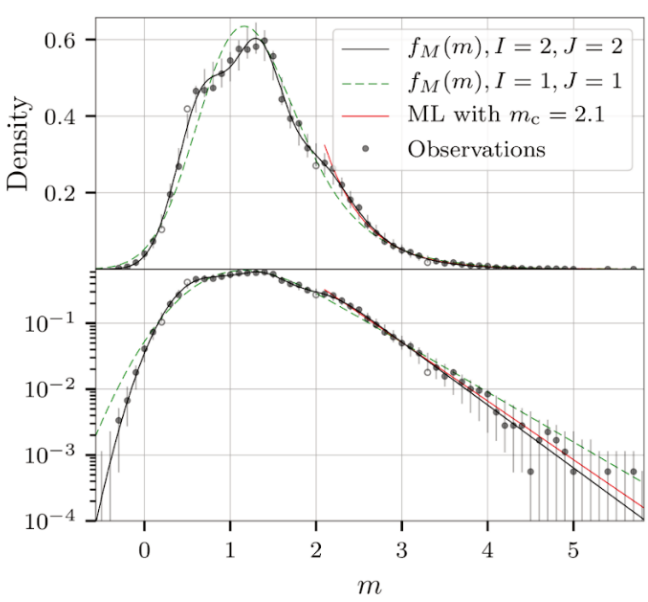

(b)

Figure 9 Observations (dots) and fitted probability density (black curves) in linear (top) and logarithmic (bottom) vertical scales (from Martinsson \& Jonsson 2018): (a) Malmberget and the orebody Dennewitz; (b) Southern California. Black curves are the proposed generalised Gutenberg-Richter law. Red curve represents the maximum likelihood estimate based on estimated magnitude of completeness using the goodness-of-fit test (Martinsson \& Jonsson 2018). Dashed green curve is the simplest distribution model (Martinsson \& Jonsson 2018)

\subsection{Tomographic models}

From the residues of solving the Swiss cheese problem, tomographic images can be created to describe the rock mass. The resolution of the tomographic images depends on the amount of data (i.e. ray coverage) and the quality of the data. Applying BEMIS auto-processing, the ray coverage is increased an order of magnitude compared to manual processing. It is a multiplicative effect of the increased number of sensors considered in the processing of the events as shown in Figure 2 and the increased number of detectable events seen in Figure 5. Additionally, the statistical approach adopted in all modules in BEMIS means that all values are associated with a measure of quality. As with all sequential processing in BEMIS, this quality measure is taken into account when constructing tomographic images of the rock mass. Tomographic images can be used to map stress changes, excavated volumes and geological variations in the rock mass and may therefore, be valuable information in the design of infrastructure, exploration and in hazard assessments in mines.

In Figure 10, we observe a slice of the estimated P-velocity along one of the orebodies in Malmberget for a certain time window. Images of the rock mass can also be generated from other parameters in the calibration such as the S-wave velocity and the energy attenuation in the rock mass (see Appendix B).

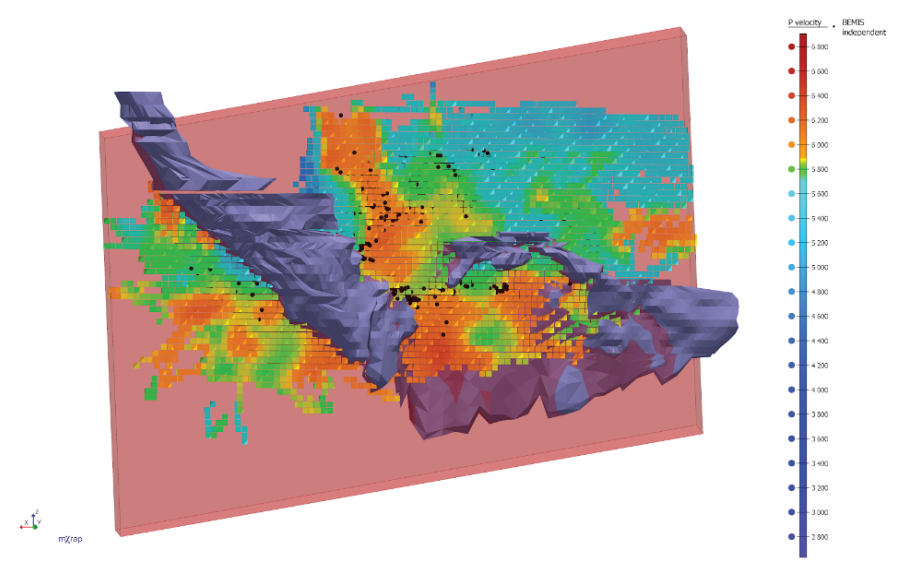

Figure 10 Example of a tomographic slice along the dip of one orebody in Malmberget mine where the black dots are recent events recorded in the slice, where shown events are mainly located in high P-velocity zones or in boundaries between high and low P-velocity zones 


\section{$4 \quad$ Automatic processing limitations}

Automatic algorithms also have limitations. The algorithms are commonly developed based on assumed operating conditions and special cases. If events are recorded that do not fulfil these conditions, automatic algorithms may fail in delivering satisfying results in rare cases. These could be, for example, if several events occur at the same time and are associated as one by the system, or if the data is corrupted. Normally, these are conditions that are equally difficult for manual processing. Fortunately, for failed events, alarms can easily be raised when automatic algorithms fail to deliver satisfying results based on numerous quality measures. Natural covariates for alarm algorithms are different quality measures that are delivered with the final result (e.g. the Deviance Information Criteria in Gelman et al. 2004) in combination with the number of waveforms included. This easily detects if a large event is problematic to process, which is of primarily importance. For smaller events, with only a handful of waveforms, it is naturally more difficult for algorithms and humans as there is little evidence provided. Fortunately, these events are of lesser importance for the mine. In BEMIS, failed events are logged for further analysis and currently these logs contain far less than one per thousand processed events. This could be compared against individual differences in rejection and acceptance shown in Appendix A and Figure A1. As opposed to manual processing, algorithmic fixes to special scenarios that do not fulfil previously assumed operating conditions will apply to all data.

\section{Conclusion}

Increasing the sensitivity of the seismic measurement system by introducing more sensors will quickly approach the limit of the capabilities of manual processing. For Malmberget and Kiruna mines only a fraction of the available data is used to locate the events. The future of mining involves a huge amount of sensors of different kinds and this future should also belong to seismic monitoring and analysis. We need more sensors to understand the rock mass and our impact on it in both small local volumes of interest and scaling up to large, mine-wide volumes. The future of seismic monitoring requires fast and accurate automatic processing of all events without human limitations.

Processing seismic data with satisfying results in a mining environment is a challenging task due to excavated volumes, complex geology, large stress changes and spatiotemporal noises. We show that this task can be solved automatically by compensating for the environment at each site, using the surrounding seismicity as calibration data. Additionally, we show that the compensation data itself can be used to analyse the rock mass using tomographic techniques.

Based on 290 blasts with known locations, the automatic processing surpasses human processing in both accuracy and precision by an order of magnitude. For mining-induced events, the algorithm detects significantly more events and resulting clusters of events are more sharply resolved. Additionally, the frequency-magnitude charts indicate improved classification of the events, e.g. the majority of the orepass noise is correctly identified, leading to a much improved catalogue for better seismological analysis than is possible with manually processed data. The inclusion of uncertainties and the use of statistical models combined with customised inference methods, means that the automatic algorithms can use significantly more data to process the events and additionally provide realistic uncertainties of the resulting parameters. These uncertainties (quality measures) are used to weight or filter the result in sequential analysis.

The automatic algorithms developed are included in a processing package for mining-induced events called BEMIS. It provides a faster, more accurate and cost-effective alternative for bulk processing of the seismic data, allowing the mine's seismic specialist to focus their attention on the largest or the most complex events and provide expert interpretation.

\section{Acknowledgement}

The authors acknowledge the financial support from Luossavaara-Kiirunavaara AB (LKAB), Sweden, and strategic innovation program STRIM, a joint venture of Vinnova, Formas and the Swedish Energy Agency. 


\section{Appendix}

This section contains additional information regarding classification of events in Section $A$ and illustrations of heterogeneous rock mass seen from the sensors at different mines in Section B.

\section{A Classification}

In Figure A1, we have used the same 500 events from Malmberget that were processed by the RMP and additionally processed by 16 professional processors. Here we compare against the RMP conducted by a larger group of individuals. From Figure 2 we can conclude that compared to RMP:

1. Each professional processor differs significantly in similarity against RMP in determining if an event should be rejected or accepted.

2. There is a significant difference between the classification results among the 16 professional processors.

3. Given a certain classification threshold, an automatic algorithm cannot be distinguished among the 16 processors.

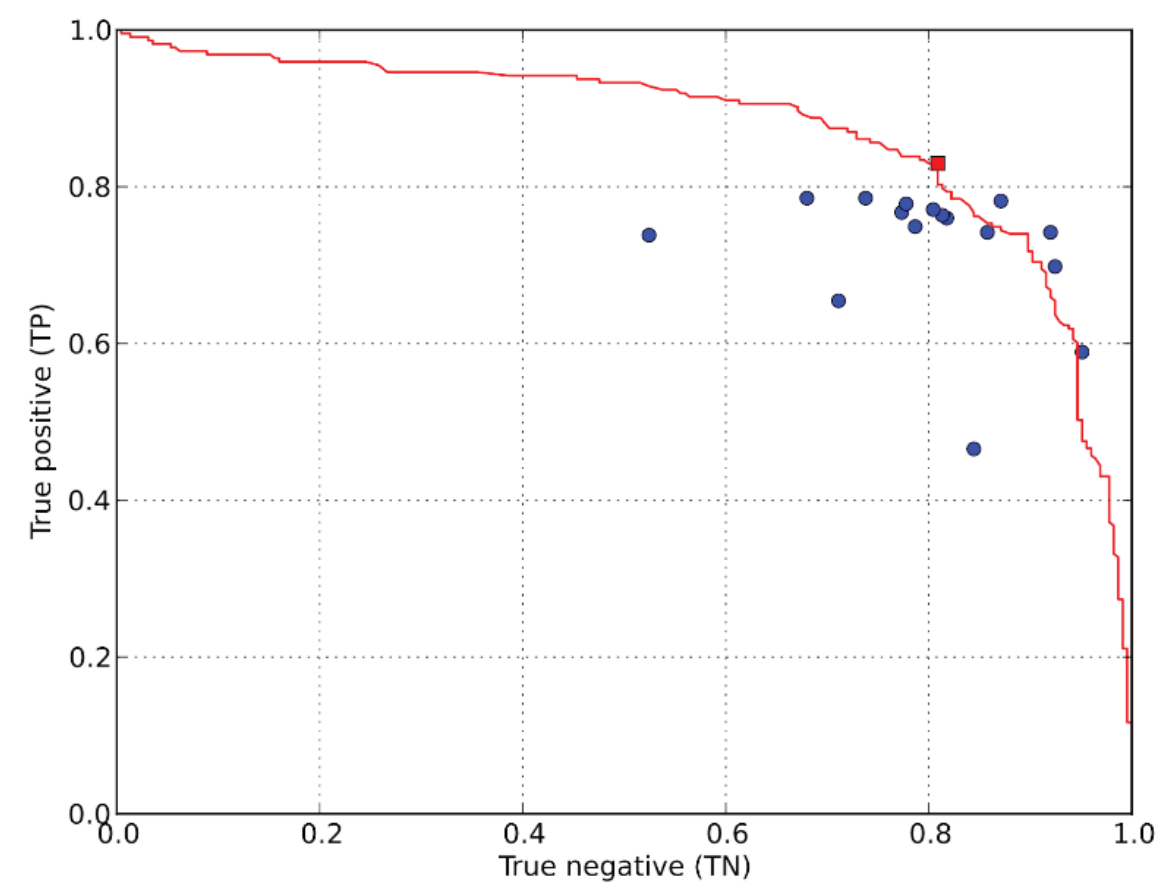

Figure A1 Receiver operating characteristic curves for the classification algorithm (red square) and 16 experienced processors (blue dots) when compared against routine manual processing (RMP) in Malmberget. The true positive (TP) factor and true negative (TN) factor are shown on different axes. A high TN factor means that we reject the events as noise similar to RMP and a high TP factor means that we accept the events as seismic events similar to RMP (where a factor 1.0 on both axes gives identical results to routine manual processing). The red curve shows the TP and TN for different classification thresholds in the classification algorithm and we select a value trying to obtain both a high TP and TN provided by the red square

In Figure A2, we compare RMP with BEMIS auto-processing with emphasis to reduce orepass noise while preserving genuine events. We observe an improved resolution using BEMIS especially around the orepasses where we are able to easily distinguish them apart and associate clusters of events to the specific orepasses shown as vertical lines. 


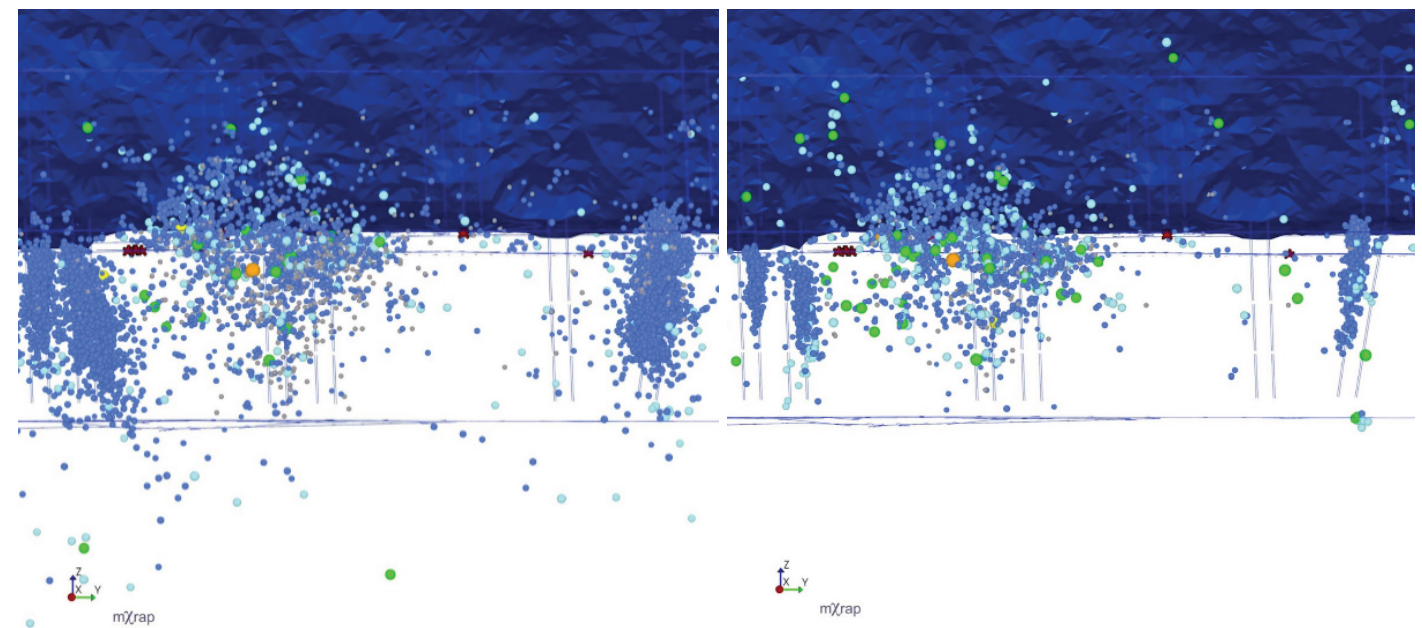

(a)

(b)

Figure A2 Comparison in Kiruna between (a) Routine manual processing and (b) BEMIS auto-processing. Orepasses are visualised as vertical grey dashed lines. As opposed to the comparison in Figure 5, using default BEMIS settings, we choose in (b) to visualise only well-located events with small uncertainty volume to emphasise this feature in BEMIS. The effect is that orepass noise is significantly reduced while preserving genuine events. The middle cluster contains one large event in orange (occurred in December 2019) and multiple aftershocks are preserved. The blue surveys are the extracted rock mass

\section{B Calibration data for each sensor}

Here we look into the internal workings of BEMIS and visualise the raw data used to solve the Swiss cheese problem for each site. Examples are shown for three different mines (Malberget, Kiruna and Garpenberg) and illustrate the heterogeneities in the rock mass. In Figure B1, we see examples of velocity and rotation as seen by different sensors. In Figure B2, we see the differences in attenuation as seen by two sensors opposite the excavated rock.

Site ID $=44$

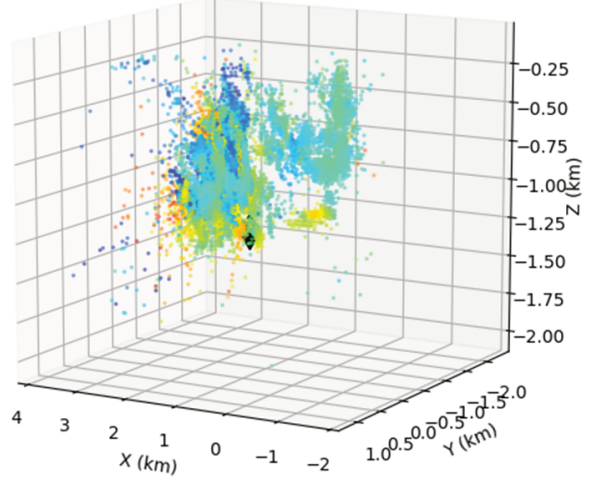

(a)
Site ID $=27$
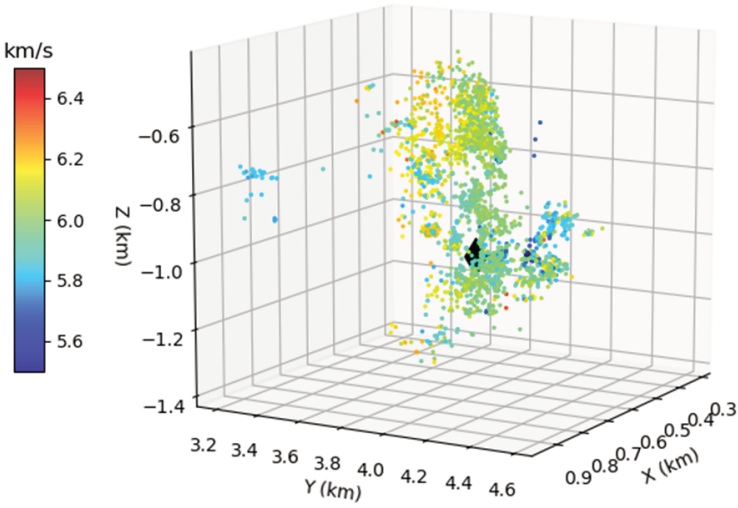

(b)

Figure B1 Examples of calibration data used to solve Swiss cheese problem: (a) P-wave velocities for sensor 44 in Malmberget; (b) Angular deviation $\theta^{c}$ (radians) of first motion in relation to homogeneous rock mass for sensor 27 in Garpenberg 


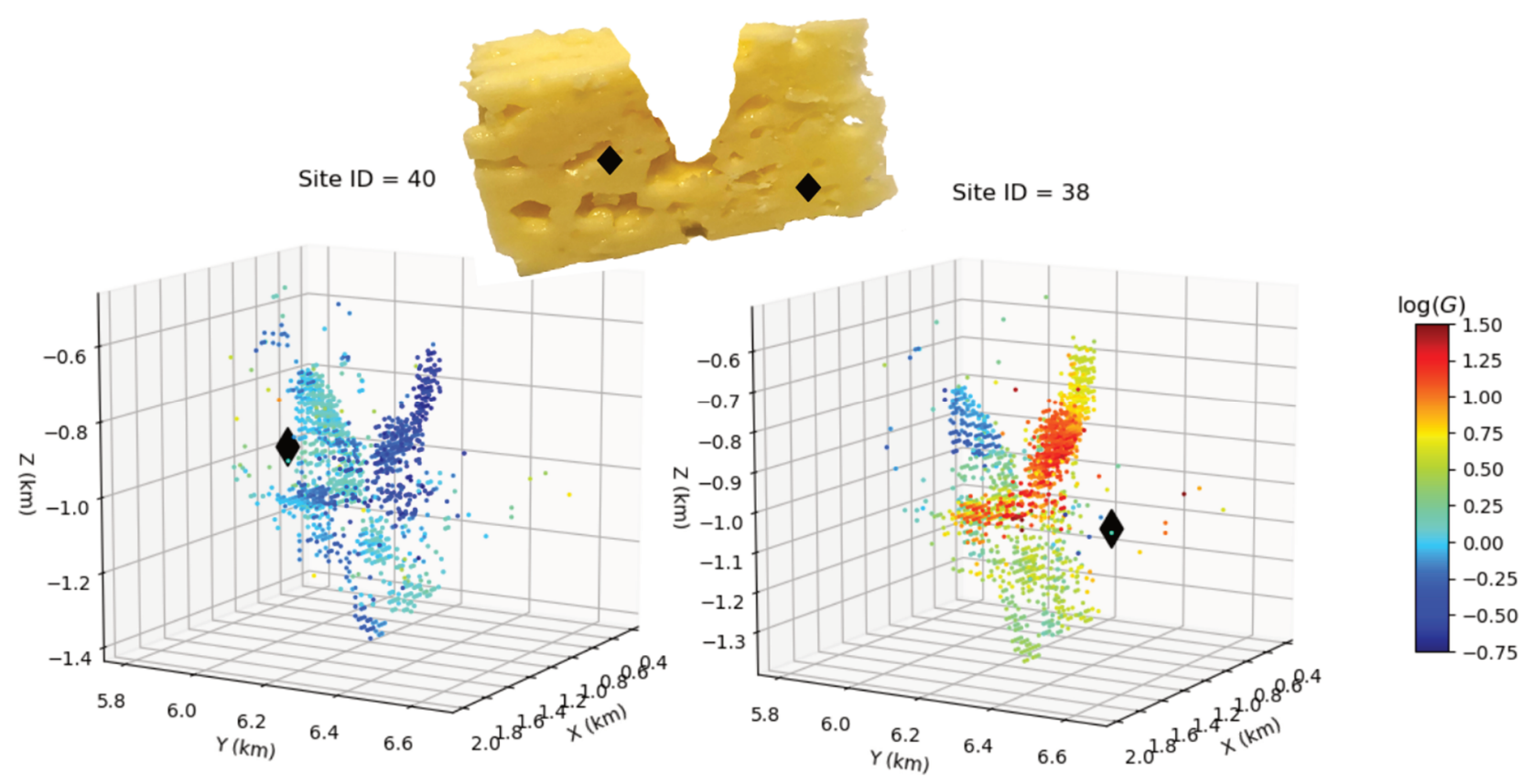

(a)

(b)

Figure B2 Example of the compensation for the expected energies between a sensor (black diamond) placed in (a) Footwall, and (b) Hanging wall, shown in the lake-ore part in the Kiruna mine. The dots are the events, coloured as a logged gain factor (including the site amplification for the sensor) in relation to the expected event energy in a homogeneous rock mass. The figures show the large energy absorption caused by the extracted rock mass in the middle of the sensors (i.e. between the 'bunny-ears' in both figures). This compensation is needed to obtain more precise event energy estimation

\section{References}

Brune, J 1970, 'Tectonic stress and the spectra of seismic shear waves from earthquakes', Journal of Geophysical Research, vol. 75, issue 26, pp. 4997-5009.

Gelman, A, Carlin, JB, Stern, HS \& Rubin, DB 2004, Bayesian Data Analysis, 2nd edn, Chapman \& Hall/CRC, London.

Harris, PH \& Wesseloo, J 2015, mXrap, version 5, computer software, Australian Centre for Geomechanics, The University of Western Australia, Perth, www.mxrap.com

Kay, S 1993, Fundamentals of Statistical Signal Processing: Estimation Theory, volume 1, Prentice Hall, Upper Saddle River.

Kruschke, J 2014, Doing Bayesian Data Analysis: A Tutorial Introduction with R, JAGS and Stan, 2nd edn, Elsevier Science, London.

Martinsson, J 2012, 'Robust Bayesian hypocentre and uncertainty region estimation: the effect of heavy-tailed distributions and prior information in cases with poor, inconsistent and insufficient arrival times', Geophysical Journal International, vol. 192, issue 3, pp. 1156-1178.

Martinsson, J \& Jonsson, A 2018, 'A new model for the distribution of observable earthquake magnitudes and applications to b-value estimation', IEEE Geoscience and Remote Sensing Letters, vol. 15, issue 6, pp. 833-837.

Martinsson, J \& Törnman, W 2019, 'Modelling the Dynamic Relationship Between Mining Induced Seismic Activity and Production Rates, Depth and Size: A Mine-Wide Hierarchical Model', Pure and Applied Geophysics, vol. 177, pp. 2619-2639.

Nordqvist, A \& Wimmer, M 2016, 'Holistic approach to study gravity flow at the Kiruna sublevel caving mine', Proceedings of the Seventh International Conference and Exhibition on Mass Mining, The Australasian Institute of Mining and Metallurgy, Carlton, pp. 401-414.

Nordström, E, Dineva, S \& Nordlund, E 2020, 'Back analysis of short-term seismic hazard indicators of larger seismic events in deep underground mines (LKAB, Kiirunavaara mine, Sweden)', Pure and Applied Geophysics, vol. 177, issue 2, pp. 763-785.

Pintelton, R \& Schoukens, J 2001, System Identification: A Frequency Domain Approach, Wiley-IEEE Press, Piscataway.

Wettainen, T \& Martinsson, J 2014, 'Estimation of future ground vibration levels in Malmberget town due to mining-induced seismic activity', Journal of the Southern African Institute of Mining and Metallurgy, Johannesburg, vol. 114, pp. 835-843.

Sjöberg, J, Dahnér, C, Malmgren, L \& Perman, F 2011, 'Forensic analysis of a rockburst event at the Kiirunavaara Mine - results and implications for the future', in D Sainsbury, R Hart, C Detournay \& M Nelson (eds), Proceedings of the 2nd International FLAC/DEM Symposium, Itasca International Inc., Minneapolis, pp. 67-74. 
Troll, VR, Weis, FA, Jonsson, E, Andersson, UB, Majidi, SA, Högdahl, K...\& Nilsson, KP 2019, 'Global Fe-O isotope correlation reveals magmatic origin of Kiruna-type apatite-iron oxide ores', Nature Communications, vol. 10, https://doi.org/ 10.1038/s41467019-09244-4

Vallejos, J \& McKinnon, S 2011, 'Correlations between mining and seismicity for re-entry protocol development', International Journal of Rock Mechanics and Mining Sciences, vol. 48, issue 4, pp. 616-625.

Vatcher, J, McKinnon, S \& Sjöberg, J 2015, 'Developing 3-D mine-scale geomechanical models in complex geological environments, as applied to the Kiirunavaara mine', Engineering Geology, vol. 36, https://doi.org/10.1016/j.enggeo.2015.07.020

Vatcher, J, McKinnon, S \& Sjöberg, J 2018, 'Rock mass characteristics and tomographic data', Rock Mechanics and Rock Engineering, vol. 51, pp. 1615-1619. 
Reliable automatic processing of seismic events: solving

W Törnman and J Martinsson

the Swiss cheese problem 